\title{
Structure of the Resource Theory of Quantum Coherence
}

\author{
Alexander Streltsov, ${ }^{1,2,3,}{ }^{*}$ Swapan Rana ${ }^{4}$ Paul Boes ${ }^{3}$ and Jens Eisert ${ }^{3}$ \\ ${ }^{1}$ Faculty of Applied Physics and Mathematics, Gdańsk University of Technology, 80-233 Gdańsk, Poland \\ ${ }^{2}$ National Quantum Information Centre in Gdańsk, 81-824 Sopot, Poland \\ ${ }^{3}$ Dahlem Center for Complex Quantum Systems, Freie Universität Berlin, D-14195 Berlin, Germany \\ ${ }^{4}$ ICFO_Institut de Ciències Fotòniques, The Barcelona Institute of Science and Technology, 08860 Castelldefels, Spain
}

(Received 24 May 2017; revised manuscript received 14 July 2017; published 5 October 2017)

\begin{abstract}
Quantum coherence is an essential feature of quantum mechanics which is responsible for the departure between the classical and quantum world. The recently established resource theory of quantum coherence studies possible quantum technological applications of quantum coherence, and limitations that arise if one is lacking the ability to establish superpositions. An important open problem in this context is a simple characterization for incoherent operations, constituted by all possible transformations allowed within the resource theory of coherence. In this Letter, we contribute to such a characterization by proving several upper bounds on the maximum number of incoherent Kraus operators in a general incoherent operation. For a single qubit, we show that the number of incoherent Kraus operators is not more than 5, and it remains an open question if this number can be reduced to 4 . The presented results are also relevant for quantum thermodynamics, as we demonstrate by introducing the class of Gibbs-preserving strictly incoherent operations, and solving the corresponding mixed-state conversion problem for a single qubit.
\end{abstract}

DOI: 10.1103/PhysRevLett.119.140402

Quantum resource theories [1,2] provide a strong framework for studying fundamental properties of quantum systems and their applications for quantum technology. The basis of any quantum resource theory is the definition of free states and free operations. Free states are quantum states which can be prepared at no additional cost, while free operations capture those physical transformations which can be implemented without consumption of resources. Having identified these two main features, one can study the basic properties of the corresponding theory, such as possibility of state conversion, resource distillation, and quantification. An important example is the resource theory of entanglement, where free states are separable states, and free operations are local operations and classical communication $[3,4]$.

In the resource theory of quantum coherence [5-9], free states are identified as incoherent states

$$
\rho=\sum_{i} p_{i}|i\rangle\langle i|,
$$

i.e., states which are diagonal in a fixed specified basis $\{|i\rangle\}$. The choice of this basis depends on the particular problem under study, and in many relevant scenarios such a basis is naturally singled out by the unavoidable decoherence [10].

The definition of free operations within the theory of coherence is not unique, and several approaches have been discussed in the literature, based on different physical (or mathematical) considerations [8]. Two important frameworks are known as incoherent [6] and strictly incoherent operations $[7,11]$, which will be denoted by IO and SIO, respectively. The characterizing feature of $\mathrm{IO}$ is the fact that they admit an incoherent Kraus decomposition; i.e., they can be written as [6]

$$
\Lambda(\rho)=\sum_{j} K_{j} \rho K_{j}^{\dagger},
$$

where each of the Kraus operators $K_{j}$ cannot create coherence individually, $K_{j}|m\rangle \sim|n\rangle$ for suitable integers $n$ and $m$. This approach is motivated by the fact that any quantum operation can be interpreted as a selective measurement in which outcome $j$ occurs with probability $p_{j}=\operatorname{Tr}\left[K_{j} \rho K_{j}^{\dagger}\right]$, and the state after the measurement is given by $K_{j} \rho K_{j}^{\dagger} / p_{j}$. An $\mathrm{IO}$ can then be interpreted as a measurement which cannot create coherence even if one applies postselection on the measurement outcomes.

Strictly incoherent operations are incoherent operations with the additional property that all $K_{i}^{\dagger}$ are also incoherent [7,11]. These operations have several desirable properties which distinguish them from the larger class IO. In particular, it has been shown that SIO is the most general class of operations which do not use coherence [11].

Other important frameworks which are discussed in the recent literature include maximally incoherent operations (MIO) [5]: this is the most general class of operations which cannot create coherence from incoherent states. It has recently been shown that this framework has maximally coherent mixed states, i.e., quantum states which are the optimal resource among all states with a given spectrum [12]. Another important class are translationally invariant operations (TIO) [13], these are quantum operations which commute with time translations $e^{-i H t}$ induced by a given 
Hamiltonian $H$. The set IO is strictly larger than TIO for nondegenerate Hamiltonians [14]. Moreover, the class TIO has found several applications in the literature, including the resource theory of asymmetry [13-15] and quantum thermodynamics [16,17]. Further approaches, also going beyond incoherent states, have been investigated recently in Refs. [18-23].

The quantification of coherence is another important research direction. Postulates for coherence quantifiers have been presented [5,6,8,24], based on earlier approaches in entanglement theory $[3,4,25]$. Operational coherence measures include distillable coherence and coherence cost $[7,26]$, which quantify optimal rates for asymptotic coherence distillation and dilution via the corresponding set of free operations. Another operational quantifier is the robustness of coherence [27,28], which is also closely related to coherence quantifiers based on interferometric visibility [29]. Distance-based coherence quantifiers have also been investigated $[6,30,31]$, and it was shown that the relative entropy of coherence is equal to the distillable coherence for the sets IO and MIO [7]. For the latter set, distillable coherence coincides with the coherence cost, which implies that the resource theory of coherence based on MIO is reversible [7]. Dynamics of coherence quantifiers under noisy evolution has also been investigated [32-37].

While initially formulated for a single particle, the framework of coherence has recently been extended to distributed scenarios [38-41]. This extension has found several applications in remote quantum protocols, including the tasks of quantum state merging $[42,43]$ and assisted coherence distillation [38,41], which has also been demonstrated experimentally very recently [44]. Coherence in multipartite systems has also been studied with respect to other types of nonclassicality such as entanglement $[21,23,30,45]$ and quantum discord [46,47].

Having identified relevant classes of free operations, it is now important to ask about a description of those operation efficient in the physical dimension. Such an efficient description seems crucial for a rigorous investigation of the corresponding resource theory. In entanglement theory, it is known that the set of local operations and classical communication is notoriously difficult to capture mathematically [48]. In this Letter, we address this question for the resource theory of coherence, focusing on the classes IO and SIO. We show that both classes admit a minimal standard form, and use these results to give a full solution for the mixed-state conversion problem via SIO, IO, and MIO. We further introduce the set of Gibbs-preserving SIO, and solve the mixed-state conversion problem for a single qubit also for these operations.

Summary of results.-A general quantum operation, acting on a Hilbert space of dimension $d$, admits a decomposition with at most $d^{2}$ Kraus operators [49] - this is the maximum Kraus rank. However, since (strictly) incoherent Kraus operators have a very specific structure, it is unclear if this result also transfers to IO and SIO [50]. In the following two statements, we provide upper bounds for the number of (strictly) incoherent Kraus operators for these operations. We refer to this minimal number as the (strictly) incoherent Kraus rank.

Theorem 1.-(Maximum number of incoherent Kraus operators for IO.) Any incoherent operation acting on a Hilbert space of dimension $d$ admits a decomposition with at most $d^{4}+1$ incoherent Kraus operators. For $d=2$ and $d=3$, this number can be improved to 5 and 39, respectively.

This theorem is a combination of Propositions 3, 4, and 5, which will be presented and discussed below. The corresponding bound for SIO is given in the following statement.

Theorem 2.-(Maximum number of strictly incoherent Kraus operators for SIO.) Any strictly incoherent operation acting on a Hilbert space of dimension $d$ admits a decomposition with at most $\min \left\{d^{4}+1, \sum_{k=1}^{d} d ! /(k-1) !\right\}$ strictly incoherent Kraus operators.

This theorem follows by combining Propositions 3 and 6. In general, it remains an open question if the bounds in Theorems 1 and 2 are tight. However, as we prove in Proposition 7, there exist SIO which require $d^{2}$ Kraus operators. This implies that the bound in Theorem 2 is tight for SIO on a single qubit.

The above theorems assist in a rigorous investigation of the resource theory of coherence, since they significantly reduce the number of free parameters for the sets IO and SIO. For a single qubit, any IO admits a decomposition into 5 incoherent Kraus operators, and for SIO this number further reduces to 4 .

We will now demonstrate the power of these results by providing a full characterization for all possible state transformations via single-qubit SIO, IO, and MIO. As we show in Sec. I of the Supplemental Material [51], a single-qubit state $\rho$ with Bloch vector $\left(r_{x}, r_{y}, r_{z}\right)^{T}$ can be converted into another single-qubit state $\sigma$ with Bloch vector $\left(s_{x}, s_{y}, s_{z}\right)^{T}$ via SIO, IO, or MIO if and only if the following inequalities are fulfilled:

$$
\begin{aligned}
s_{x}^{2}+s_{y}^{2} & \leq r_{x}^{2}+r_{y}^{2}, \\
s_{z}^{2} & \leq 1-\frac{1-r_{z}^{2}}{r_{x}^{2}+r_{y}^{2}}\left(s_{x}^{2}+s_{y}^{2}\right) .
\end{aligned}
$$

For a given initial Bloch vector $\left(r_{x}, r_{y}, r_{z}\right)^{T}$, these inequalities completely characterize the achievable region for the final Bloch vectors $\left(s_{x}, s_{y}, s_{z}\right)^{T}$. The achievable region is symmetric under rotations around the $z$ axis and corresponds to a cylinder with radius $\left(r_{x}^{2}+r_{y}^{2}\right)^{1 / 2}$ and height $2 r_{z}$ with ellipsoids attached at the top and the bottom. In Fig. 1 we show the projection of the achievable region into the $x-z$ plane for four initial states. The proof of Eqs. (3) in Sec. I of the Supplemental Material [51] makes use of our result that any single-qubit SIO can be decomposed into four strictly incoherent Kraus operators, see Theorem 2 and discussion 


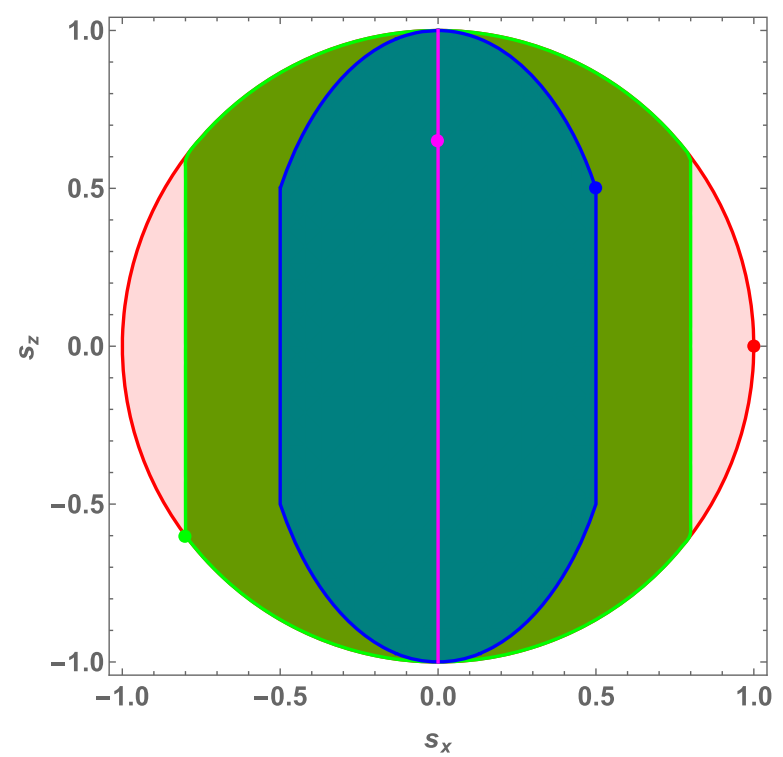

FIG. 1. Achievable region for single-qubit SIO, IO, and MIO. Colored areas show the projection of the achievable region in the $x-z$ plane for initial Bloch vectors $(0.5,0,0.5)^{T}$ [blue], $(-0.8,0,-0.6)^{T}$ [green], and $(1,0,0)^{T}$ [red]. Note that the last two states are pure. The magenta line corresponds to the achievable region of an incoherent state with Bloch vector $(0,0,0.65)^{T}$.

below Proposition 4 for the general form of these operators. Alternatively, the form of the achievable region can be proven using results in Refs. [18-20]. We also note that for pure states the conversion problem has been completely solved for SIO [7] and IO [45].

As a second application for our results, we investigate strictly incoherent operations which preserve a given incoherent state $\tau$, i.e.,

$$
\Lambda(\tau)=\tau .
$$

The motivation for this constraint originates from quantum thermodynamics. In particular, if $\tau=e^{-\beta H} / \operatorname{Tr}\left[e^{-\beta H}\right]$ is the Gibbs state of a system with Hamiltonian $H$, then the condition (4) is known to hold for thermal operations $[16,17,52]$. For a nondegenerate Hamiltonian $H$ thermal operations cannot create coherence in the eigenbasis of $H$, and conditions for state transformations under these operations and the role of coherence therein have been extensively studied in Refs. [53-55]. The most general quantum operations that fulfill Eq. (4) are known as Gibbspreserving operations, and it has been shown that such operations can create coherence [56]. Here, we contribute to this discussion by introducing the set of Gibbs-preserving SIO, and giving a full solution for the mixed-state conversion problem on a single qubit. As an example, in Fig. 2 we show the achievable region in the $x-z$ plane for an initial state $\rho$ with Bloch vector $\boldsymbol{r}=(0.5,0,0.5)^{T}$, and the preserved Gibbs state $\tau$ has the Bloch vector $\boldsymbol{t}=$ $(0,0,-0.2)^{T}$. Note that the achievable region is convex

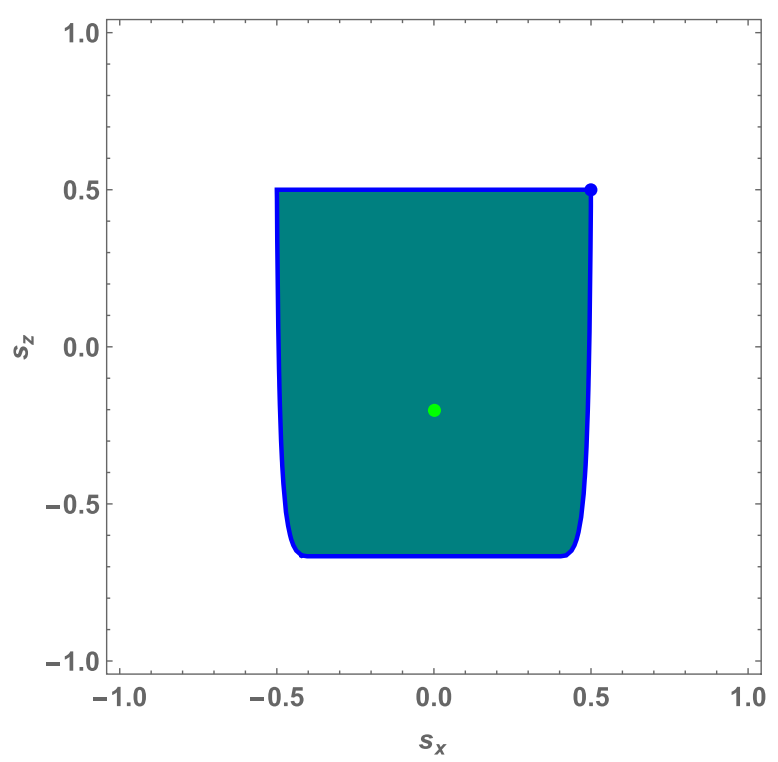

FIG. 2. Achievable region [blue area] of Gibbs-preserving SIO on a single qubit. The initial state has the Bloch vector $\boldsymbol{r}=$ $(0.5,0,0.5)^{T}$ [blue dot] and the preserved Gibbs state has the Bloch vector $\boldsymbol{t}=(0,0,-0.2)^{T}$ [green dot].

and symmetric under rotations around the $z$ axis. We refer to Sec. II of the Supplemental Material [51] for more details and further examples.

This discussion clearly demonstrates how Theorems 1 and 2 lead to deep insights on the structure of the resource theory of quantum coherence. In particular, they lead to a full solution of the state conversion problem under singlequbit SIO, IO, and MIO. These results have clear relevance within the resource theory of coherence, and also extend to other related fields, including quantum thermodynamics.

In the remainder of this Letter we will present statements which are needed for proving the aforementioned theorems, and provide further detailed discussion of the results.

Bounds from Choi-Jamiotkowski isomorphism.-We will now present bounds for IO and SIO arising from the Choi-Jamiołkowski isomorphism between a quantum operation $\Lambda$ and the corresponding Choi state

$$
\rho_{\Lambda}=(\Lambda \otimes \mathrm{id})\left(\Phi_{d}^{+}\right),
$$

where $\Phi_{d}^{+}=d^{-1} \sum_{i, j=0}^{d-1}|i, i\rangle\langle j, j|$ is a maximally entangled state of dimension $d^{2}$. The rank of the Choi state is the Kraus rank, which is the smallest number of (not necessarily incoherent) Kraus operators.

Proposition 3.--(Bounds originating from the Choi state.) Any (strictly) incoherent operation acting on a Hilbert space of dimension $d$ admits a decomposition with at most $d^{4}+1$ (strictly) incoherent Kraus operators.

The proof can be found in Sec. III of the Supplemental Material [51]. It is interesting to note that the proof of the above proposition does not use the fact that the Kraus operators are incoherent. Thus, the proposition is not 
limited to IO, but also applies to SIO. Moreover, it also applies to separable operations, i.e., operations of the form

$$
\Lambda_{\mathrm{s}}(\rho)=\sum_{i}\left(A_{i} \otimes B_{i}\right) \rho\left(A_{i}^{\dagger} \otimes B_{i}^{\dagger}\right) .
$$

Any separable operation can be decomposed into (at most) $d^{4}+1$ product Kraus operators $A_{i} \otimes B_{i}$, where $d=d_{A} d_{B}$ is the dimension of the total system. The same is true for separable quantum-incoherent operations [41], i.e., operations of the form (6) with incoherent operators $B_{i}$, and separable incoherent operations where both $A_{i}$ and $B_{i}$ are incoherent [41]. For single-qubit IO and SIO the upper bound in Proposition 3 gives 17 operators. As we will show in the following, this number can be significantly reduced.

Bounds from the structure of (strictly) incoherent operations.-We will now provide improved bounds which explicitly make use of the structure of IO and SIO. For this, we will first consider incoherent operations on a single qubit; i.e., the corresponding Hilbert space has dimension $d=2$. The following proposition shows that any singlequbit IO can be decomposed into 5 incoherent Kraus operators with a simple structure.

Proposition 4.-(Incoherent operations on qubits.) Any incoherent operation acting on a single qubit admits a decomposition with at most 5 incoherent Kraus operators. A canonical choice of the operators is given by the set

$\left\{\left(\begin{array}{cc}a_{1} & b_{1} \\ 0 & 0\end{array}\right),\left(\begin{array}{cc}0 & 0 \\ a_{2} & b_{2}\end{array}\right),\left(\begin{array}{cc}a_{3} & 0 \\ 0 & b_{3}\end{array}\right),\left(\begin{array}{cc}0 & b_{4} \\ a_{4} & 0\end{array}\right),\left(\begin{array}{cc}a_{5} & 0 \\ 0 & 0\end{array}\right)\right\}$,

where $a_{i}$ can be chosen real, while $b_{i} \in \mathbb{C}$. Moreover, it holds that $\sum_{i=1}^{5} a_{i}^{2}=\sum_{j=1}^{4}\left|b_{j}\right|^{2}=1$ and $a_{1} b_{1}+a_{2} b_{2}=0$.

We refer to Sec. IV of the Supplemental Material [51] for the proof. We also note that the same techniques can be applied to single-qubit SIO, in which case the number of strictly incoherent operators reduces to 4 . The corresponding general form of strictly incoherent Kraus operators can be given as

$$
\left\{\left(\begin{array}{cc}
a_{1} & 0 \\
0 & b_{1}
\end{array}\right),\left(\begin{array}{cc}
0 & b_{2} \\
a_{2} & 0
\end{array}\right),\left(\begin{array}{cc}
a_{3} & 0 \\
0 & 0
\end{array}\right),\left(\begin{array}{cc}
0 & 0 \\
a_{4} & 0
\end{array}\right)\right\},
$$

where $a_{i}$ are real and $\sum_{i=1}^{4} a_{i}^{2}=\sum_{j=1}^{2}\left|b_{j}\right|^{2}=1$. A more general bound for SIO for arbitrary dimensions will be given below.

It is important to note that the proofs of Propositions 3 and 4 are fundamentally different: while the argument leading to Proposition 3 is based on the Choi-Jamiołkowski isomorphism and Caratheodory's theorem, the proof of Proposition 4 makes explicit use of the structure of IO. In the next step, we will extend Proposition 4 to arbitrary dimension.

Proposition 5.-(IO for $d$-dimensional systems.) Any incoherent operation for a quantum systems of dimension $d$ admits a decomposition with at most $d\left(d^{d}-1\right) /(d-1)$ incoherent Kraus operators.

We refer to Sec. V of the Supplemental Material [51] for the proof. For single-qubit IO Proposition 5 gives us 6 incoherent operators as an upper bound. As we have already seen in Proposition 4, this number can be reduced to 5. For qutrits we obtain 39 Kraus operators, while the bound in Proposition 3 gives 82 Kraus operators. For dimensions larger than 3, Proposition 3 always gives a better bound.

We will now see how the tools presented above can be applied to study the structure of SIO. By Proposition 3, any SIO admits a decomposition into (at most) $d^{4}+1$ strictly incoherent Kraus operators. As we will show in the following, for small dimensions this number can be significantly reduced.

Proposition 6.-(SIO for $d$-dimensional systems.) Any strictly incoherent operation acting on a Hilbert space of dimension $d$ admits a decomposition with at most $\sum_{k=1}^{d} d ! /(k-1)$ ! strictly incoherent Kraus operators.

The proof of the proposition can be found in Sec. VI of the Supplemental Material [51]. Note that the bound in this proposition is below $d^{4}+1$ for $d \leq 5$. For larger dimensions $d^{4}+1$ gives a better bound. For $d=2$ we see that any single-qubit SIO admits a decomposition into 4 strictly incoherent Kraus operators. This was already discussed below Proposition 4. As we will show in the following, this bound is tight.

Proposition 7.-(Lower bound.) For a Hilbert space of dimension $d$, there exist strictly incoherent operations which cannot be implemented with fewer than $d^{2}$ Kraus operators.

The proof of the proposition can be found in Sec. VII of the Supplemental Material [51]. Note that for $d=2$ the bounds in Propositions 6 and 7 coincide: any single-qubit SIO can be decomposed into 4 strictly incoherent Kraus operators, and some single-qubit SIO require 4 Kraus operators in their decomposition.

Conclusions. - In this work we have studied the structure of the resource theory of quantum coherence, focusing in particular on the structure of incoherent and strictly incoherent operations. We have shown that any (strictly) incoherent operation can be written with at most $d^{4}+1$ (strictly) incoherent Kraus operators, where $d$ is the dimension of the Hilbert space under study. For small dimensions this number can be significantly reduced. For a single qubit any IO can be decomposed into 5 incoherent Kraus operators, while any SIO admits a decomposition into 4 strictly incoherent Kraus operators. While the latter bound is tight, the tightness of the other bounds remains an open question.

Our results assist in the systematic investigation of the resource theory of coherence due to the significant reduction of unknown parameters. We have applied our results to solve the mixed-state conversion problem for single-qubit $\mathrm{SIO}, \mathrm{IO}$, and MIO. We further introduced the class of 
Gibbs-preserving strictly incoherent operations and also solved the corresponding mixed-state conversion problem for a single qubit. A natural next step would be to consider single-qubit incoherent operations applied on one subsystem of a bipartite quantum state. Such multipartite scenarios have been previously studied in Refs. [38-41], and the results presented in this Letter provide a strong framework for their further investigation. Another important question which is left open in this work is the relation of Gibbspreserving strictly incoherent operations to thermal operations. We expect that further results in this direction will be presented in the near future, exploring Gibbs-preserving strictly incoherent operations in relation to recent works on quantum thermodynamics [16,17,53-55] and extending them to other notions of quantum coherence [8].

Finally, our results also transfer to other related concepts, including translationally invariant operations, which are relevant in the resource theory of asymmetry and quantum thermodynamics. Recalling that TIO is a subset of IO, the results presented in this Letter also give bounds on decompositions of TIO into incoherent Kraus operators. Thus, numerical simulations and optimizations over all these classes now also become feasible, at least for small dimensions.

We thank Gerardo Adesso, Dario Egloff, Martin Plenio, Thomas Theurer, Henrik Wilming, and, especially, Andreas Winter for discussions. We acknowledge financial support by the Alexander von Humboldt-Foundation, the National Science Center in Poland (POLONEZ UMO-2016/21/P/ ST2/04054), EU grants OSYRIS (ERC-2013-AdG Grant No. 339106), TAQ (ERC CoG Grant No. 307498) and QUIC (H2020-FETPROACT-2014 Grant No. 641122), the Spanish MINECO grant FISICATEAMO (FIS2016-79508$\mathrm{P}$ ), the Severo Ochoa Programme (SEV-2015-0522), MINECO CLUSTER (ICFO15-EE-3785), the Generalitat de Catalunya (2014 SGR 874 and CERCA/Program), the Fundació Privada Cellex, the DFG (CRC 183), and Studienstiftung des Deutschen Volkes.

Note added.-Upon completion of this manuscript, a related work has appeared [57].

*streltsov.physics@gmail.com

[1] M. Horodecki and J. Oppenheim, Int. J. Mod. Phys. B 27, 1345019 (2013).

[2] B. Coecke, T. Fritz, and R. W. Spekkens, Inf. Comput. 250, 59 (2016).

[3] V. Vedral, M. B. Plenio, M. A. Rippin, and P. L. Knight, Phys. Rev. Lett. 78, 2275 (1997).

[4] R. Horodecki, P. Horodecki, M. Horodecki, and K. Horodecki, Rev. Mod. Phys. 81, 865 (2009).

[5] J. Åberg, arXiv:quant-ph/0612146.

[6] T. Baumgratz, M. Cramer, and M. B. Plenio, Phys. Rev. Lett. 113, 140401 (2014).

[7] A. Winter and D. Yang, Phys. Rev. Lett. 116, 120404 (2016).
[8] A. Streltsov, G. Adesso, and M. B. Plenio, arXiv: 1609.02439 [Rev. Mod. Phys. (to be published)].

[9] K. Ben Dana, M. García Díaz, M. Mejatty, and A. Winter, Phys. Rev. A 95, 062327 (2017).

[10] W. H. Zurek, Rev. Mod. Phys. 75, 715 (2003).

[11] B. Yadin, J. Ma, D. Girolami, M. Gu, and V. Vedral, Phys. Rev. X 6, 041028 (2016).

[12] A. Streltsov, H. Kampermann, S. Wölk, M. Gessner, and D. Bruß, arXiv:1612.07570 .

[13] G. Gour and R. W. Spekkens, New J. Phys. 10, 033023 (2008).

[14] I. Marvian, R. W. Spekkens, and P. Zanardi, Phys. Rev. A 93, 052331 (2016).

[15] J. A. Vaccaro, F. Anselmi, H. M. Wiseman, and K. Jacobs, Phys. Rev. A 77, 032114 (2008).

[16] M. Lostaglio, K. Korzekwa, D. Jennings, and T. Rudolph, Phys. Rev. X 5, 021001 (2015).

[17] M. Lostaglio, D. Jennings, and T. Rudolph, Nat. Commun. 6, 6383 (2015).

[18] E. Chitambar and G. Gour, Phys. Rev. Lett. 117, 030401 (2016).

[19] E. Chitambar and G. Gour, Phys. Rev. A 94, 052336 (2016).

[20] E. Chitambar and G. Gour, Phys. Rev. A 95, 019902(E) (2017).

[21] N. Killoran, F. E. S. Steinhoff, and M. B. Plenio, Phys. Rev. Lett. 116, 080402 (2016).

[22] T. Theurer, N. Killoran, D. Egloff, and M. B. Plenio, arXiv:1703.10943 .

[23] B. Regula, M. Piani, M. Cianciaruso, T. R. Bromley, A. Streltsov, and G. Adesso, arXiv:1704.04153.

[24] F. Levi and F. Mintert, New J. Phys. 16, 033007 (2014).

[25] M. B. Plenio and S. Virmani, Quantum Inf. Comput. 7, 1 (2007).

[26] X. Yuan, H. Zhou, Z. Cao, and X. Ma, Phys. Rev. A 92, 022124 (2015).

[27] C. Napoli, T. R. Bromley, M. Cianciaruso, M. Piani, N. Johnston, and G. Adesso, Phys. Rev. Lett. 116, 150502 (2016).

[28] M. Piani, M. Cianciaruso, T. R. Bromley, C. Napoli, N. Johnston, and G. Adesso, Phys. Rev. A 93, 042107 (2016).

[29] T. Biswas, M. García Díaz, and A. Winter, Proc. R. Soc. A 473, 20170170 (2017).

[30] A. Streltsov, U. Singh, H. S. Dhar, M. N. Bera, and G. Adesso, Phys. Rev. Lett. 115, 020403 (2015).

[31] S. Rana, P. Parashar, and M. Lewenstein, Phys. Rev. A 93, 012110 (2016).

[32] T. R. Bromley, M. Cianciaruso, and G. Adesso, Phys. Rev. Lett. 114, 210401 (2015).

[33] I. A. Silva, A. M. Souza, T. R. Bromley, M. Cianciaruso, R. Marx, R. S. Sarthour, I. S. Oliveira, R. Lo Franco, S. J. Glaser, E. R. deAzevedo, D. O. Soares-Pinto, and G. Adesso, Phys. Rev. Lett. 117, 160402 (2016).

[34] A. Mani and V. Karimipour, Phys. Rev. A 92, 032331 (2015).

[35] M. García-Díaz, D. Egloff, and M. B. Plenio, Quantum Inf. Comput. 16, 1282 (2016).

[36] Z. Puchała, L. Pawela, and K. Życzkowski, Phys. Rev. A 93, 062112 (2016).

[37] P. Zanardi, G. Styliaris, and L. Campos Venuti, Phys. Rev. A 95, 052306 (2017). 
[38] E. Chitambar, A. Streltsov, S. Rana, M. N. Bera, G. Adesso, and M. Lewenstein, Phys. Rev. Lett. 116, 070402 (2016).

[39] E. Chitambar and M.-H. Hsieh, Phys. Rev. Lett. 117, 020402 (2016).

[40] J. M. Matera, D. Egloff, N. Killoran, and M. B. Plenio, Quantum Sci. Technol. 1, 01LT01 (2016).

[41] A. Streltsov, S. Rana, M. N. Bera, and M. Lewenstein, Phys. Rev. X 7, 011024 (2017).

[42] A. Streltsov, E. Chitambar, S. Rana, M. N. Bera, A. Winter, and M. Lewenstein, Phys. Rev. Lett. 116, 240405 (2016).

[43] M. Horodecki, J. Oppenheim, and A. Winter, Nature (London) 436, 673 (2005).

[44] G. Xiang, in Nonlinear Optics (Optical Society of America, Washington, DC, 2017), p. NW4A.20.

[45] H. Zhu, Z. Ma, Z. Cao, S.-M. Fei, and V. Vedral, arXiv:1704.01935 [Phys. Rev. A (to be published)].

[46] J. Ma, B. Yadin, D. Girolami, V. Vedral, and M. Gu, Phys. Rev. Lett. 116, 160407 (2016).

[47] K. Modi, A. Brodutch, H. Cable, T. Paterek, and V. Vedral, Rev. Mod. Phys. 84, 1655 (2012).

[48] E. Chitambar, D. Leung, L. Mančinska, M. Ozols, and A. Winter, Commun. Math. Phys. 328, 303 (2014).
[49] M. A. Nielsen and I. L. Chuang, Quantum Computation and Quantum Information, 10th ed. (Cambridge University Press, Cambridge, England, 2010).

[50] Clearly, any IO and SIO admits a decomposition into (at most) $d^{2}$ Kraus operators, where $d$ is the dimension of the Hilbert space. However, it is unclear if such a minimal decomposition will have the desired (strictly) incoherent structure.

[51] See Supplemental Material at http://link.aps.org/ supplemental/10.1103/PhysRevLett.119.140402 for technical proofs and further discussion.

[52] D. Janzing, P. Wocjan, R. Zeier, R. Geiss, and T. Beth, Int. J. Theor. Phys. 39, 2717 (2000).

[53] M. Horodecki and J. Oppenheim, Nat. Commun. 4, 2059 (2013).

[54] F. Brandão, M. Horodecki, N. Ng, J. Oppenheim, and S. Wehner, Proc. Natl. Acad. Sci. U.S.A. 112, 3275 (2015).

[55] P. Ćwikliński, M. Studziński, M. Horodecki, and J. Oppenheim, Phys. Rev. Lett. 115, 210403 (2015).

[56] P. Faist, J. Oppenheim, and R. Renner, New J. Phys. 17, 043003 (2015).

[57] H.-L. Shi, X.-H. Wang, S.-Y. Liu, W.-L. Yang, Z.-Y. Yang, and H. Fan, arXiv:1705.00785. 